\title{
Diacronie
}

Studi di Storia Contemporanea

$\mathrm{N}^{\circ} 28,4 \mid 2016$

La voce del silenzio

\section{Arnault Skornicki, Jérôme Tournadre, La nouvelle histoire des idées politiques}

\section{Elisa Grandi}

\section{Q OpenEdition \\ 1 Journals}

\section{Edizione digitale}

URL: http://journals.openedition.org/diacronie/4510

DOI: $10.4000 /$ diacronie. 4510

ISSN: 2038-0925

\section{Editore}

Association culturelle Diacronie

\section{Notizia bibliografica digitale}

Elisa Grandi, «Arnault Skornicki, Jérôme Tournadre, La nouvelle histoire des idées politiques », Diacronie [Online], $N^{\circ} 28,4$ | 2016, Messo online il 29 décembre 2016, consultato il 23 septembre 2020. URL: http://journals.openedition.org/diacronie/4510; DOI : https://doi.org/10.4000/diacronie.4510 


\section{Diacronie}

N. 28 | 4|2016 La voce del silenzio: intelligence, spionaggio e conflitto nel XX secolo

19/

\section{PANORAMICA: Francia 2015}

Émilien RUIZ, Elisa GRANDI, Francesca SANNA*

LE GAC, Julie, OLLIVIER, Anne-Laure, SPINA, Raphaël, WIEVIORKA, Olivier (dir.), La France en chiffres de 1870 à nos jours, Paris, Perrin, 2015, 850 pp. ${ }^{1}$

a cura di Emilien RUIZ

traduzione di Elisa GRANDI

La France en chiffre è innanzitutto un'impresa documentaria. Il primo obiettivo del lavoro, in effetti è di offrire un panorama statistico il più possibile esaustivo della Francia nel XX secolo. Un libro dunque imprescindibile, che permette, in poco tempo, di reperire informazioni fondamentali sui diversi aspetti dello Stato e della società francese nel XX secolo. Organizzato in 5 macro aree (demografia, economia, società, politica, guerre e crisi), raccoglie dati talvolta pubblicati da organismi pubblici (l'INSEE, l'INED, etc.), talvolta recuperati attraverso altri lavori storiografici. I primi capitoli sono consacrati alle principali questioni economiche e demografiche, anche attraverso una prospettiva comparata rispetto ad altri paesi. In seguito vengono riportate informazioni su tematiche riguardanti le elezioni politiche, le categorie socioprofessionali e i principali conflitti militari che hanno visto impegnata la Francia: dalla guerra del 1870 alla guerra di Algeria, passando per le guerre coloniali e i conflitti mondiali. L'opera ha dunque un indiscusso valore di documentazione e si presta ad essere un ottimo strumento di lavoro per gli studenti. Tuttavia, vi si possono riscontrare alcuni limiti, sia nella contestualizzazione delle fonti, sia per quanto riguarda la loro tracciabilità. In alcuni casi, in effetti, l'istituto che ha prodotto i dati viene citato, ma senza fornire altre indicazioni che permettano al lettore di rinvenire gli studi utilizzati.

\footnotetext{
${ }^{1}$ L'articolo è tratto da una versione più ampia apparsa sulla rivista «La Vie des Idées», il 31 agosto 2015.
} 
Inoltre, non è sempre ben indicato se le tabelle siano prese direttamente dalla fonte, $o$ siano, piuttosto, il frutto di un'elaborazione degli autori a partire da più fonti e materiali. Risulta infine un problema il fatto che non venga fatto accenno a come le statistiche vennero costruite e ai dibattiti che si produssero di volta in volta davanti alla costruzioni di serie storiche sul lungo periodo. Infine, manca ogni riferimento al libero accesso, in linea, di alcune fra le statistiche riportate. Sarebbe stato invece utile, per il lettore, sapere che alcune statistiche pubbliche sono disponibili su Gallica ${ }^{2}$. Ci si sarebbe potuto aspettare anche un riferimento diretto alle statistiche apparse sul Centre de recherche historique 3 e sul sito dell'INSEE4.

Queste osservazioni non tolgono nulla alla pertinenza e all'utilità della pubblicazione, che costituisce sicuramente un riferimento fondamentale per studenti e insegnanti.

QUENNOUËLLE-CORRE, Laure, La place financière de Paris au XXe siècle. Des ambitions contrariées, Paris, Comité pour l'histoire économique et financière de la France-IGPDE, 2015, 512 pp.

traduzione di Elisa GRANDI

L’opera traccia il percorso del mercato finanziario di Parigi dalla Prima guerra mondiale alla fine degli anni Ottanta, seguendo una prospettiva internazionale. Sono ricostruite le diverse fasi di affermazione e declino della borsa di Parigi rispetto alle altre borse internazionali, attraverso un'analisi sia dei principali attori che vi operavano, sia dell'insieme di pratiche e regole che organizzavano il mercato dei titoli. In particolare, l'autrice si concentra sulle banche e sulla loro attività di immissione di titoli nel mercato finanziario, così come sull'evoluzione del loro prestigio internazionale. Vengono ugualmente studiati i principali intermediari finanziari e le regole in cui operavano. Il terzo angolo di analisi è costituito dal ruolo dello Stato e delle altre istituzioni di controllo, come la banca centrale.

Al centro dello studio vi sono i fattori che hanno influito sul rilievo internazionale del mercato di Parigi e l'analisi delle ambizioni degli attori che vi operavano. Articolandosi su tre periodi (1914-1939; 1940-1978; 1979-1988), il libro descrive i vincoli alle strategie messe in campo dagli attori e i risultati raggiunti. Uno studio

\footnotetext{
${ }^{2}$ URL: < http://gallica.bnf.fr/ark:/12148/cb34349577d/date > [consultato il 23 aprile 2016]. 3 URL: < http://acrh.revues.org/2890 > [consultato il 23 aprile 2016].

${ }_{4}$ URL: < http://www.insee.fr/fr/service/bibliotheque/tableaux_sgf/tableaux.asp?domaine=rec $>$ [consultato il 23 aprile 2016].
} 
approfondito di molteplici fondi documentari ha permesso un resoconto dettagliato tanto delle riforme messe in atto, quanto dei circuiti di credito ufficiale e secondario.

\section{SKORNICKI, Arnault, TOURNADRE, Jérôme, La nouvelle histoire des idées politiques, Paris, La Découverte, 2015, 128 pp.}

a cura di Elisa GRANDI

A partire dagli anni Settanta, le metodologie per la ricostruzione della storia delle idee politiche sono cambiate radicalmente. Con "la nuova storia delle idee politiche", si afferma la necessità di superare uno studio delle idee dei grandi classici del pensiero politico in quanto fonte di riflessione su una determinata problematica, per giungere a un'analisi del contesto storico in cui determinate teorie sono state pensate. Secondo questo approccio, quindi, l'idea politica, «non esiste indipendentemente dell'uso, storicamente situato, in cui si è prodotta». Partendo dalla necessità di ricostruire questi dibattiti e diffonderli al pubblico francofono, il libro ne ripercorre le fasi principali in cinque capitoli, che affrontano ognuno una corrente storiografica ed epistemologica ascrivibile alla nuova storia delle idee politiche. Vi trovano posto in particolare alcuni approcci propri della tradizione anglosassone (la scuola di Cambridge, affrontata nel primo capitolo), ma anche la semantica storica tedesca (secondo capitolo). Sono allo stesso tempo descritti alcuni progetti metodologici nati in seno alla stessa tradizione francese, come la storia sociale delle idee politiche, che affronta lo studio degli intellettuali dalla prospettiva di una sociologia storica, concentrandosi sugli intermediari culturali e sui meccanismi di ricezione delle idee. Senza pretesa di esaustività, questo libro permette dunque di inquadrare questi approcci sottolineandone un tratto comune: la volontà di studiare le configurazioni sociali e storiche in cui le idee politiche vengono elaborate.

PASSAQUI, Jean-Philippe, Les voyages forment l'ingénieur. Les houillères du Centre-Midi de la France (1851-1873), Paris, Garnier, 2015, 564 pp.

a cura di Francesca SANNA

Les voyages formes les ingénieurs è un saggio di Jean Philippe Passaqui - studioso che vanta una vasta produzione scientifica sui temi della storia economica e dell'industria francese. L'opera si pone all'incrocio di diverse branche della storiografia, dalla storia d'impresa a quella delle istituzioni educative, ma si ritrova pienamente 
integrato nella storiografia francese delle professioni. Il testo intende apportare, però, un nuovo contributo allo studio del milieu di formazione del Corpo degli Ingenieurs des Mines nella seconda metà del XIX secolo, attingendo alla fonte dei carnets de voyage e dei mémoires degli allievi dell'Ecole des Mines di Parigi.

Gli stage, soprattutto nel Centre e nel Midi francesi, sono considerati una miniera di informazioni: vi si trovano descrizioni di un sistema produttivo costantemente affetto da sfavorevoli condizioni geologiche e morfologiche e, nel ventennio 1850-1873, riflessioni sulla crisi produttiva, la contrazione dei mercati e la divaricazione tecnologica fra le Compagnie. Perciò, attraverso i mémoires, Passaqui intende anche capire se allievi e ingegneri fossero in grado di giungere ad una visione globale dei problemi dell'impresa mineraria francese.

Il risultato è un testo ricchissimo di informazioni, accompagnate da accurate riproduzioni dei disegni degli allievi-ingegneri e da curiose suggestioni aneddotiche. In questa abbondanza emerge però con chiarezza il rilievo dei cambiamenti tecnologici che interessarono la regione nella seconda metà del XIX secolo. Alcuni allievi-ingegneri se ne resero conto durante il loro viaggio ed infatti Passaqui termina il suo studio affermando che «au délà de l'aspect purement technique de leurs descriptions, se dessin bien sous la plume des élèves l'organisation de l'action technique»5, anticipando quello che poi sarà lo sviluppo dell'industria carbonifera francese nei decenni successivi.

5 PASSAQUI, Jean-Philippe, Les voyages forment l'ingénieur. Les houillères du Centre-Midi de la France (1851-1873), Paris, Garnier, 2015, p. 502. 


\section{* Gli autori}

Elisa Grandi si è laureata in Storia all'Università di Bologna nel 2005, ha ottenuto la specializzazione in Storia del Mondo contemporaneo nello stesso ateneo, nel 2007. È alumna del Collegio Superiore dell'Università di Bologna. È stata Visiting Student alla Duke University (2009), Visiting Scholar alla New School of Social Research (2010), borsista Eiffel-Excellence presso il laboratorio SEDET di Parigi (2011) e Attaché de Recherche all'Université Paris Diderot (2011-2012). Sta completando un dottorato di storia economica all'Universita Paris Diderot, in cotutela con l'Università di Bologna, sui piani di sviluppo finanziati dalla Banca Mondiale in Europa e America Latina negli anni Cinquanta e Sessanta. Dal 2012 lavora come Senior Researcher nell'Equipex DFIH (Données Financières Historiques) della Paris School of Economics e dal 2014 insegna Storia della globalizzazione all'Université Paris 7 - Denis Diderot.

URL: < http://www.studistorici.com/2010/12/07/elisa_grandi/ >

Francesca Sanna ha conseguito la Laura triennale in Storia presso l'Università degli studi di Milano nel 2012 con una tesi sul profilo storico-economico dell'industria estrattiva sarda del secondo dopoguerra. Ha poi ottenuto nel 2014 il doppio diploma (Laurea Magistrale e Master 2 Recherche) del «Master italo-francese di Storia e Civiltà Comparate», in cotutela fra l'Université Paris 7 - Denis Diderot e l'Università degli Studi di Bologna con una tesi sull'applicazione dei sistemi di scientific management nell'industria mineraria. Dopo un Master di II livello in «Conservazione gestione e valorizzazione del patrimonio industriale» all'Università di Padova, è attualmente dottoranda all'Université Paris 7 - Denis Diderot con un contratto dottorale «USPC Paris Sorbonne Cité - volet international».

URL: < http://www.studistorici.com/progett/autori/\#Sanna_Francesca >

Émilien Ruiz è maître de conference presso l'Université de Lille. Ha insegnato metodologia della ricerca storica all'EHESS e storia contemporanea e informatica per le scienze storiche all'Università di Paris 7 - Denis Diderot, dove si è addottorato. Dal 2012 collabora ai corsi di Paul André Rosental all'Istituto di Scienze Politiche di Parigi. È cofondatore (con Franziska Heimburger) de La Boite à Outils des Historiens, blog di informazione scientifica sugli strumenti informatici per le scienze storiche; gestisce inoltre il blog Devenir historien-ne, sulla metodologia della ricerca storica e sulla storiografia.

URL: < http://www.studistorici.com/2010/12/o7/emilien_ruiz/ > 


\section{Per citare questo articolo:}

RUIZ, Émilien, GRANDI, Elisa, SANNA, Francesca, «Panoramica: Francia 2015», Diacronie. Studi di Storia Contemporanea : La voce del silenzio: intelligence, spionaggio e conflitto nel XX secolo, 29/12/2016,

URL:< http://www.studistorici.com/2016/12/29/francia_numero_28/ >

Diacronie Studi di Storia Contemporanea $\vartheta$ www.diacronie.it

Risorsa digitale indipendente a carattere storiografico. Uscita trimestrale. redazione.diacronie@hotmail.it

Comitato di redazione: Jacopo Bassi - Luca Bufarale - Antonio César Moreno Cantano - Deborah Paci - Fausto Pietrancosta - Alessandro Salvador - Matteo Tomasoni - Luca Zuccolo

Diritti: gli articoli di Diacronie. Studi di Storia Contemporanea sono pubblicati sotto licenza Creative Commons 3.0. Possono essere riprodotti e modificati a patto di indicare eventuali modifiche dei contenuti, di riconoscere la paternità dell'opera e di condividerla allo stesso modo. La citazione di estratti è comunque sempre autorizzata, nei limiti previsti dalla legge. 